Gastroenterologe 2009 · 4:499-500 DOI 10.1007/s11377-009-0365-4

Online publiziert: 17. Oktober 2009

(c) Springer Medizin Verlag 2009

M.P. Manns ${ }^{1} \cdot$ H.E. Blum ${ }^{2} \cdot$ U. Hopt ${ }^{3}$

${ }^{1}$ Gastroenterologie, Hepatologie und Endokrinologie, Medizinische Hochschule Hannover

${ }^{2}$ Abteilung Innere Medizin II, Medizinische Universitätsklinik Freiburg

${ }^{3}$ Abteilung Allgemein- und Viszeralchirurgie, Klinik und Poliklinik für Chirurgie, Universitätsklinikum Freiburg

\title{
Leber- und Pankreastransplantation
} Aktueller Stand und Perspektiven

Leber- und Pankreastransplantationen sind als solide Organtransplantationen im klinischen Alltag etabliert. Etwa 700 bis 800 Lebertransplantationen werden pro Jahr in Deutschland durchgeführt. Die chirurgische Technik hat sich dabei in den letzten Jahren nicht wesentlich verändert.

Ein großes Problem bleibt die Diskrepanz zwischen Transplantationskandidaten, d. h. dem Bedarf für eine Lebertransplantation und verfügbaren Spenderorganen. Seit einigen Jahren ist in Deutschland das in Amerika eingeführte (MELD)-System für die Organallokation übernommen worden. Der MELDScore kann einfach errechnet werden, basierend auf Bilirubin, Gerinnung und Nierenfunktion. Die Erfahrungen der letzten Jahre haben aber gezeigt, dass hierdurch immer kränkere Patienten transplantiert werden und somit die Ergebnisse nicht besser, sondern eher schlechter werden.

\section{( Das Problem der Wartezeit lässt sich prinzipiell durch die Möglichkeit der Leberlebensspende reduzieren}

Deshalb ist es wichtig, auch für die nicht direkt in der Transplantationsmedizin tätigen Ärzte einmal die Veränderungen der Situation für Lebertransplantationen nach Einführung des MELD-Systems darzustellen. Einige Erkrankungen gelten als „Standard Exceptions“, so das hepatozel- luläre Karzinom (HCC). Das HCC entwickelt sich in der Regel auf dem Boden einer Leberzirrhose und gehört zu den am stärksten zunehmenden Lebertumoren weltweit. Die Lebertransplantation stellt für Frühstadien in der Tat eine kurative Perspektive dar. Die richtige Auswahl der Patienten ist jedoch für das optimale Ergebnis essenziell, ebenso wie die Behandlung des HCC während der Wartezeit bis zur Transplantation („Bridging“).

\section{( Heute ist die Leber- transplantation auch bei Hepatitis-B-Infektion möglich}

Das Problem der Wartezeit lässt sich prinzipiell durch die Möglichkeit der Leberlebensspende („living donor liver transplantation") reduzieren. Diese stellt vor allem chirurgisch eine besondere Herausforderung dar. Bei Kindern ist die Leberlebensspende durch ein Elternteil oder andere Verwandte etabliert. Deshalb sterben Kinder kaum noch auf der Warteliste. Bei Erwachsenen ist die Lebertransplantation dagegen chirurgisch und medizinisch weiterhin eine besondere Herausforderung. $\mathrm{Zu}$ den wesentlichen Fortschritten in der Transplantationsmedizin bei Lebertransplantation gehört die Prophylaxe und auch Therapie der Hepatitis-B-Virus (HBV)-Reinfektion. Während früher die Hepatitis B eine Lebertransplantation-Kontraindikation war, ist eine Lebertransplantation heute bei He- 
patitis B problemlos möglich. Problematisch bleiben hingegen die chronische $\mathrm{He}$ patitis-C-Virus (HCV)-Infektion und ihre Endstadien, da es bisher keine Möglichkeiten gibt, die HCV-Reinfektion zu verhindern. Gleichzeitig ist die Therapie der rekurrierenden Hepatitis $\mathrm{C}$ bisher unbefriedigend.

Anders als die Lebertransplantation ist die Pankreastransplantation nur an wenigen Zentren etabliert. Sie wird in der Regel bei Diabetespatienten mit diabetischer Nephropathie zusammen mit einer Nierentransplantation durchgeführt. Die Betreuung dieser Patienten ist außerordentlich komplex. Es ist deshalb nicht zuletzt auch für den niedergelassenen Gastroenterologen, der nicht regelmäßig Nierentransplantierte und Diabetespatienten betreut, wichtig, dass er über den aktuellen Stand der Pankreastransplantationen informiert ist und die Indikationen, die Prognose sowie die Grundzüge der Betreuung dieser Patienten kennt.

Die Herausgeber dieser Ausgabe von „Der Gastroenterologe“ zum Schwerpunktthema „Leber- und Pankreastransplantation“ hoffen, dass die Beiträge aus der Transplantationsmedizin abdomineller Organe Ihr Interesse finden und für die Betreuung Ihrer Patienten nützlich sein werden.

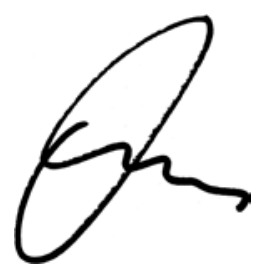

M.P. Manns

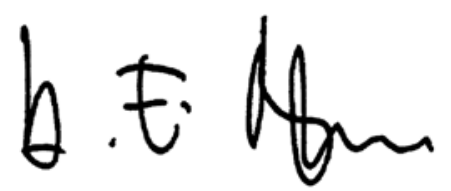

H.E. Blum

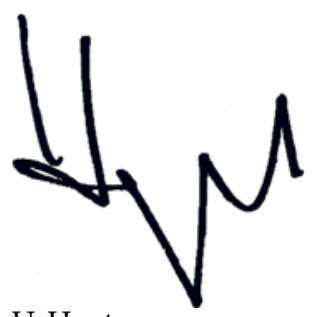

U. Hopt

\section{Korrespondenzadressen}

\section{Prof. Dr. M.P. Manns}

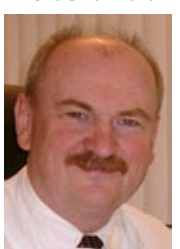

Gastroenterologie, Hepatologie und Endokrinologie, Medizinische Hochschule Hannover Carl-Neuberg-Str. 1, 30623 Hannover Manns.michael@mh-hannover.de

\section{Prof. Dr. Dr. h. c. mult. H.E. Blum}

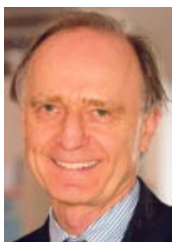

Abteilung Innere Medizin II, Medizinische Universitätsklinik, Hugstetter Str. 55, 9106 Freiburg hubert.blum@uniklinikfreiburg.de

\section{Prof. Dr. Dr. h. c. U. Hopt}

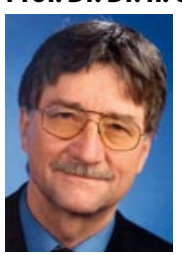

Abteilung Allgemein- und Viszeralchirurgie, Klinik und Poliklinik für Chirurgie, Universitätsklinikum, Hugstetter Straße 55, 79106 Freiburg ulrich.hopt@uniklinik-freiburg.de
Der Gastroenterologe bietet umfassende und aktuelle Beiträge zu interessanten Themenschwerpunkten aus allen Bereichen der Gastroenterologie und Hepatologie.

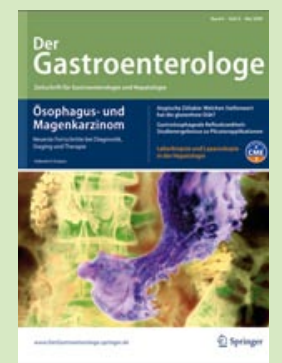

Themenschwerpunkte 2009

- 1/09 Dünndarm

- 2/09 Autoimmune Lebererkrankungen

- 3/09 Ösophagus- und Magenkarzinom

- 4/09 Viszeralmedizin-komplexe Krankheitsbilder aus hepatologischer und chirurgischer Sicht

- 5/09 Viszeralmedizin-komplexe Krankheitsbilder aus gastroenterologischer und chirurgischer Sicht

- 6/09 Leber und Pankreastransplantation

\section{Themenschwerpunkte 2010}

- 1/10 Hereditäre gastroenterologische Erkrankungen

- 2/10 NASH \& co

- 3/10 Rationaler Einsatz der Bildgebung in der Gastroenterologie

- 4/10 Interdisziplinäre Gastroenterologie

- 5/10 Proktologie

- 6/10 Gastrointestinale Onkologie

(Änderungen vorbehalten)

www.DerGastroenterologe.springer.de 